\title{
Disentangling Transient Charge Density and Metal-Ligand Covalency in Photoexcited Ferricyanide with Femtosecond Resonant Inelastic Soft X-ray Scattering
}

Raphael M. Jay, ${ }^{\dagger}$ Jesper Norell, ${ }^{\ddagger}$ Sebastian Eckert, ${ }^{\dagger, \S}$ Markus Hantschmann, ${ }^{\S}$ Martin Beye, ${ }^{\S}, \| \odot$ Brian Kennedy, ${ }^{\S}$ Wilson Quevedo, ${ }^{\S}$ William F. Schlotter, ${ }^{\perp}$ Georgi L. Dakovski, ${ }^{\perp}$ Michael P. Minitti, ${ }^{\perp}$ Matthias C. Hoffmann, ${ }^{\perp}$ Ankush Mitra,,${ }^{\perp} \bullet$ Stefan P. Moeller, ${ }^{\perp}$ Dennis Nordlund, ${ }^{\#}$ Wenkai Zhang,

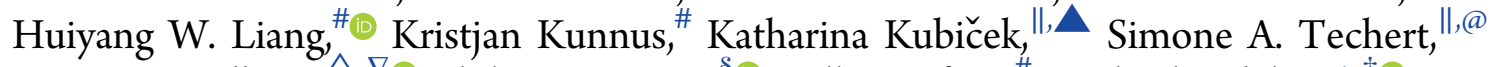
Marcus Lundberg, $\triangle, \nabla_{\odot}$ Philippe Wernet, ${ }^{\S \odot}$ Kelly Gaffney, ${ }^{\#}$ Michael Odelius, ${ }^{*}, \ddagger$ and Alexander Föhlisch ${ }^{*}, \dagger, \S$

${ }^{\dagger}$ Institut für Physik und Astronomie, Universität Potsdam, 14476 Potsdam, Germany

${ }^{\ddagger}$ Department of Physics, Stockholm University, Albanova University Center, 10691 Stockholm, Sweden

${ }^{\S}$ Institute for Methods and Instrumentation for Synchrotron Radiation Research, Helmholtz-Zentrum Berlin für Materialien und Energie GmbH, 12489 Berlin, Germany

"DESY Photon Science, 22607 Hamburg, Germany

${ }^{\perp}$ LCLS, SLAC, Menlo Park, California 94025, United States

\#PULSE Institute, SLAC, Menlo Park, California 94025, United States

${ }^{\circledR}$ Institute for X-ray Physics, Göttingen University, 37077 Göttingen, Germany

$\triangle$ Department of Chemistry - Ángström Laboratory, Uppsala University, 75121 Uppsala, Sweden

${ }^{\nabla}$ Department of Biotechnology, Chemistry and Pharmacy, Università di Siena, 53100 Siena, Italy

Supporting Information

ABSTRACT: Soft X-ray spectroscopies are ideal probes of the local valence electronic structure of photocatalytically active metal sites. Here, we apply the selectivity of timeresolved resonant inelastic X-ray scattering at the iron L-edge to the transient charge distribution of an optically excited charge-transfer state in aqueous ferricyanide. Through comparison to steady-state spectra and quantum chemical calculations, the coupled effects of valence-shell closing and ligand-hole creation are experimentally and theoretically disentangled and described in terms of orbital occupancy, metal-ligand covalency, and ligand field splitting, thereby extending established steady-state concepts to the excited-state domain. $\pi$-Back-donation is found to be mainly determined by the metal site occupation, whereas the ligand hole instead influences $\sigma$-donation. Our results demonstrate how ultrafast resonant inelastic X-ray scattering can help characterize local charge distributions around catalytic metal centers in short-lived charge-transfer excited states, as a step toward future

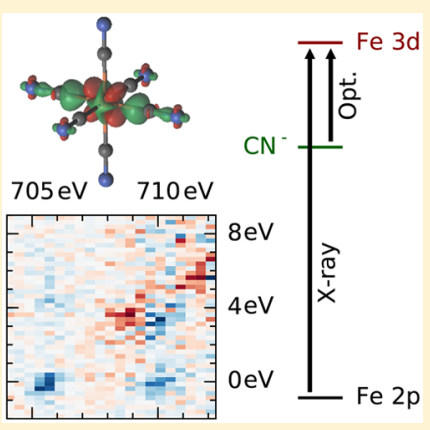
rationalization and tailoring of photocatalytic capabilities of transition-metal complexes.

$\mathrm{T}$ ransition-metal (TM) complexes play a crucial role in photocatalytic processes relevant for a fundamental chemical understanding as well as research on solar fuels. ${ }^{1}$ In the latter, catalysts based on the widely abundant and nontoxic iron $(\mathrm{Fe})$ are of particular value. ${ }^{2}$ Especially charge-transfer excited states of $\mathrm{Fe}$ complexes have attracted considerable scientific interest, as the optically induced redistribution of valence charge density can be exploited in light-harvesting applications. ${ }^{3}$ For that purpose, an understanding of how transient charge densities around the metal center govern the ability of TM complexes to facilitate chemical reactions is critical. Specifically, the localization of an electronic charge deficiency or surplus described through orbital occupancy and metal-ligand covalency has to be characterized and rationalized. Yet, disentangling these properties in real time during photochemical reactions has proven to be spectroscopically challenging.

In recent years, resonant inelastic X-ray scattering ( $\mathrm{RIXS}^{4}$ ) at metal L-edges has been established as a powerful probe of the local valence electronic structure in $3 \mathrm{~d}$ TM compounds. ${ }^{5-8}$ With the underlying $2 \mathrm{p} \rightarrow 3 \mathrm{~d}$ core-excitation of the inelastic scattering process, occupied as well as unoccupied valence

Received: May 4, 2018

Accepted: June 11, 2018

Published: June 11, 2018 
orbitals around the central metal are locally accessed, thereby probing both orbital occupancy and metal-ligand covalency. Particularly in conjunction with advanced quantum chemical simulations, the coupled effects of $\sigma$-donation and $\pi$-backdonation have been disentangled and quantified under steadystate conditions. ${ }^{6}$ With the high brilliance and time-resolution of free electron lasers (FELs), the selectivity of RIXS can also be applied to complexes in transient configurations. ${ }^{9-12}$ Femtosecond (fs) RIXS at metal L-edges can thus probe the relevant molecular properties to describe transient changes of charge density following an optical excitation and characterize in novel detail the excited-state electronic structure.

In this work, we demonstrate how time-resolved RIXS is able to retrieve previously inaccessible details of a chargetransfer excited state of the prototypical TM complex ferricyanide $\left[\mathrm{Fe}(\mathrm{III})(\mathrm{CN})_{6}\right]^{3-}$ in aqueous solution. As prime examples of octahedral back-donating complexes, ferricyanide and its reduced counterpart ferrocyanide $\left[\mathrm{Fe}(\mathrm{II})(\mathrm{CN})_{6}\right]^{4-}$ have been targets of various steady-state ${ }^{6,13-20}$ and timeresolved $^{21-25}$ studies. Here, we investigate the $400 \mathrm{~nm}$ ligandto-metal charge-transfer (LMCT) excitation in ferricyanide. ${ }^{14}$ Previous time-resolved studies have used infrared (IR) spectroscopy $^{21,25}$ and valence photoemission ${ }^{24,25}$ to primarily investigate the relaxation mechanism of the LMCT state, where the ligand-centric character of IR spectroscopy and the restriction of photoemission to occupied orbitals provide an incomplete characterization of the valence electronic structure of the LMCT excited state. In an approximative one-electron picture, the LMCT state can be described as a dipole-allowed excitation that increases the ground state (GS) $t_{2 g}^{5}$ occupation of ferricyanide (Figure $1 \mathrm{a}$ ) to $\mathrm{t}_{2 \mathrm{~g}}^{6}$ (Figure $1 \mathrm{~b}$ ). Locally at the $\mathrm{Fe}$ site, the LMCT state therefore exhibits the same formal $3 \mathrm{~d}$ closed-shell configuration $\left(t_{2 g}^{6}\right)$ as ferrocyanide (Figure 1c). In difference to ferrocyanide, however, the excitation also opens a ligand-hole in the $t_{1 u}$ manifold of ligand-derived orbitals ${ }^{14}$ $\left(\mathrm{Lt}_{1 \mathrm{u}}\right.$, see Figure $\left.\mathrm{lb}\right)$. The in-total six $\mathrm{Lt}_{1 \mathrm{u}}$ orbitals comprise three $\sigma$ - and three $\pi$-derived ligand orbitals that may mix. Thus, the relative $\sigma$ - and $\pi$-character contributions of the ligand-hole and their influence on $\sigma$-donation and $\pi$-backdonation cannot be predicted a priori. To observe both changes in valence occupancy as well as the implications of the ligand-hole on the transient electronic structure, we therefore compare the Fe L-edge RIXS spectrum of the LMCT state, detected at fs delays of our optical-pump X-ray-probe measurement, to steady-state RIXS spectra of both ferri- and ferrocyanide in their electronic GSs.

The general layout of the experimental station and details of the soft X-ray beamline have been described in previous publications. $^{26,27}$ Details on the experimental parameters can be found in the Supporting Information. The time-resolved RIXS data of ferricyanide shown in Figure $1 \mathrm{a}, \mathrm{b}$ are recorded by scanning the incident X-ray energy and pump-probe delay, while collecting the scattered X-rays. The ferricyanide GS RIXS spectrum in panel a is acquired by integrating over the negative delays up to $-70 \mathrm{fs}$. The main RIXS features in the measured range have previously been assigned and analyzed by Kunnus et al. ${ }^{6}$ and Norell et al. ${ }^{28}$ Within this study, we therefore focus on spectral features that have been previously identified as fingerprints of the LMCT state ${ }^{28}$ and thus are of particular value for its characterization. The corresponding RIXS features of GS ferricyanide are marked in Figure 1a and denoted according to the electronic occupations of frontier orbitals in the RIXS final states. Despite the highly multi-
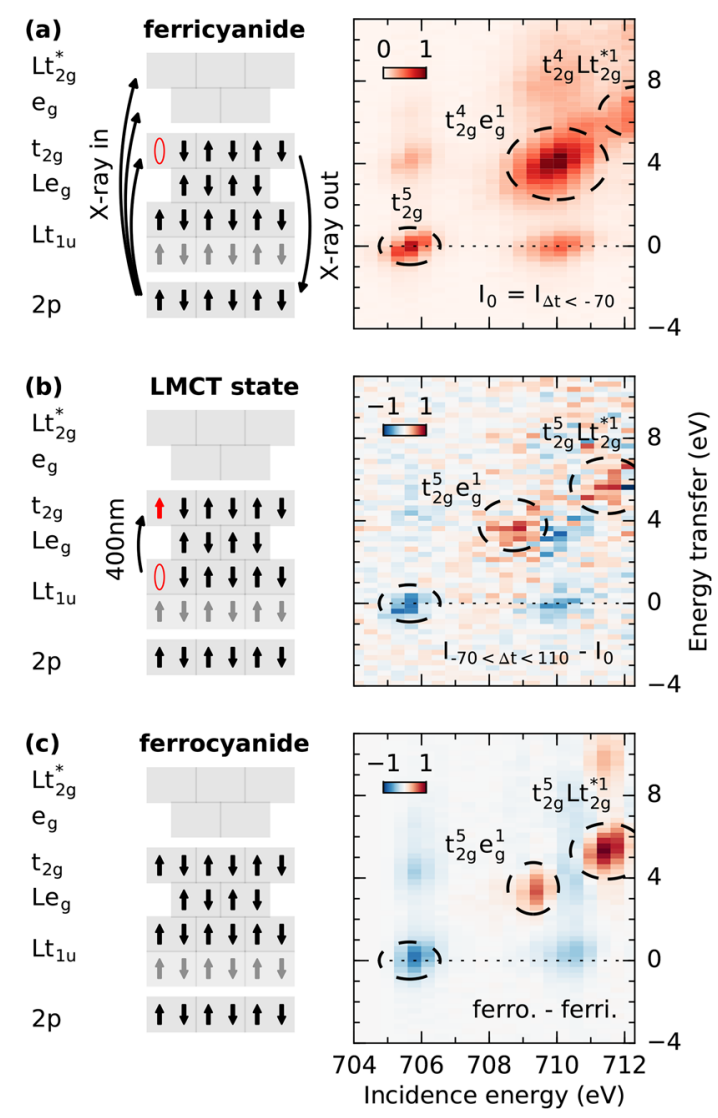

Figure 1. (a) Valence electronic occupation and schematic RIXS process together with the RIXS map of ferricyanide acquired by integrating up to $-70 \mathrm{fs}$ delay. Characteristic RIXS features are encircled and denoted according to the assigned final state occupations. (b) Valence electronic occupation of the LMCT state and difference map in the range of -70 to $110 \mathrm{fs}$. (c) Valence electronic occupation of ferrocyanide and static difference map between ferro- and ferricyanide (data taken from Kunnus et al. ${ }^{6}$ ), qualitatively reproducing the transients detected in the time-resolved measurements.

configurational nature of the involved states, they can be nominally assigned within a single-electron picture. ${ }^{6,28}$ The three features result from core-excitation into the three different (partially) unoccupied valence orbitals $\left(t_{2 g}, e_{g}\right.$, and $\mathrm{Lt}_{2 \mathrm{~g}}^{*}$ ), with varying energy transfers due to (in)elastic scattering from the $t_{2 g}$ orbitals. First, Fe $2 p \rightarrow t_{2 g}$ excitation (705.6 eV incidence energy) causes multiple scattering features indicative of the open-shell $t_{2 g}^{5}$ configuration of GS ferricyanide, with the elastic peak ( $0 \mathrm{eV}$ energy transfer) carrying the most intensity. Second, the Fe $2 \mathrm{p} \rightarrow \mathrm{e}_{\mathrm{g}}$ excitation (710 eV incidence energy) exhibits significant decay into the $\mathrm{t}_{2 \mathrm{~g}}^{4} \mathrm{e}_{\mathrm{g}}^{1}$ final state $(4 \mathrm{eV}$ energy transfer). Because of its metal-centered dd-character, it is of particular interest as a measure of the ligand field splitting (10Dq) between the $t_{2 g}$ and $e_{g}$ levels. ${ }^{5}$ This excitation is dipoleforbidden through direct optical spectroscopy yet uniquely accessible through RIXS. Third, Fe $2 \mathrm{p} \rightarrow \mathrm{Lt}_{2 \mathrm{~g}}^{*}$ excitation (signal beyond $712 \mathrm{eV}$ incidence energy) shows decay into the $\mathrm{t}_{2 \mathrm{~g}}^{4} \mathrm{Lt}_{2 \mathrm{~g}}^{* 1}$ final state ( $7 \mathrm{eV}$ energy transfer). Because of its metalto-ligand charge-transfer (MLCT) character, it directly probes the relative strength of back-donation into the ligand $\pi^{*}$ system. $^{6}$

Following the optical excitation, the early transient spectrum (integrated between -70 and $110 \mathrm{fs}$ ) corresponding to the 
LMCT state is shown in Figure 1b. Negative delays are included to account for the time-resolution of $\sim 180 \mathrm{fs}$. The short integration range is chosen to avoid spectral overlap with potential secondary species. ${ }^{24}$ The data is displayed as a difference taken with respect to the map in Figure 1a after normalizing to the number of FEL shots included in the respective integrated delay ranges. In terms of final state energies, the LMCT spectrum shows strong similarity to the static difference spectrum between ferro- and ferricyanide shown in Figure 1c. The static difference is generated from the steady-state spectra of Kunnus et al. ${ }^{6}$ after normalizing each map to its respective edge-jump (difference of post-edge intensity at $729 \mathrm{eV}$ and below any pre-edge features at 704 $\mathrm{eV}$ ). The striking similarity between the LMCT state and ferrocyanide has previously been observed with time-resolved photoemission spectroscopy in terms of binding energies of $\mathrm{Fe}$ 3d-derived orbitals. ${ }^{24,25}$ The ferrocyanide closed-shell configuration therefore constitutes a good first-order approximation of the valence electronic structure of the LMCT state from an iron-centric perspective. The static difference verifies how the valence-shell-closing of the $t_{2 g}$ orbitals in the LMCT state quenches the Fe $2 \mathrm{p} \rightarrow \mathrm{t}_{2 \mathrm{~g}}$ core-excitation, seen as a bleach of the ferricyanide $t_{2 g}^{5}$ final state in the transient map of the LMCT state (Figure 1b) and previously predicted as a spectroscopic fingerprint of the LMCT state. ${ }^{28}$ By comparison to ferrocyanide ${ }^{6}$ or computational predictions of the LMCT spectrum, ${ }^{28}$ the remaining marked transient RIXS features of the LMCT state can now be assigned to $\mathrm{t}_{2 \mathrm{~g}}^{5} \mathrm{e}_{\mathrm{g}}^{1} \mathrm{dd}$ character (709 eV incidence energy, $4 \mathrm{eV}$ energy transfer) and $\mathrm{t}_{2 \mathrm{~g}}^{5} \mathrm{Lt}_{2 \mathrm{~g}}^{* 1}$ MLCT character (711.5 eV incidence energy, $5 \mathrm{eV}$ energy transfer).

Despite the similarities between the LMCT state and ferrocyanide, differences in terms of width and position of the $t_{2 g}^{5} e_{g}^{1}$ final state can be observed, which can be assumed to result from the ligand-hole. Figure $2 \mathrm{a}$ shows the partial

(a) Incidence energy (eV)
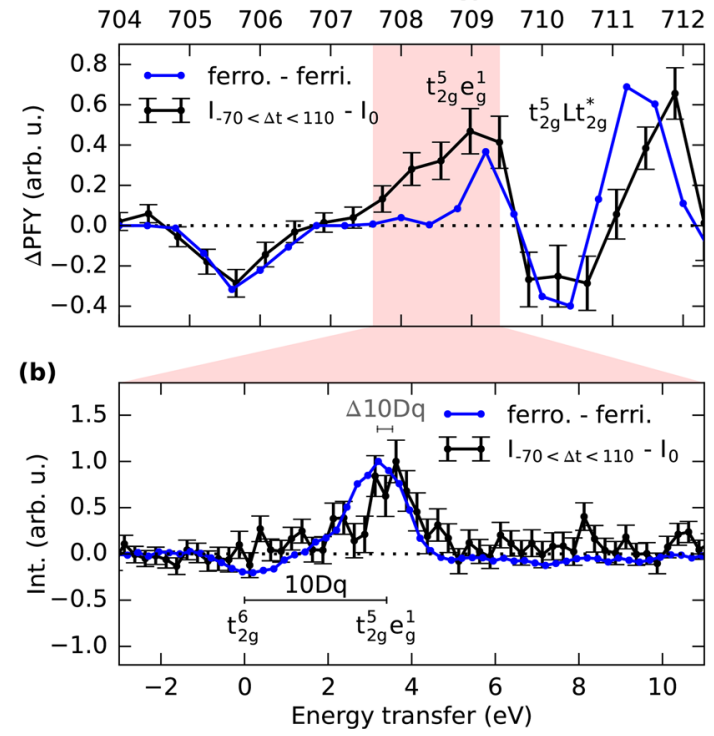

Figure 2. (a) Difference PFYs of the LMCT state and ferrocyanide with respect to ferricyanide generated by integration of the RIXS maps in Figure $1 b, c$ over energy transfers in the range of $2-7.5 \mathrm{eV}$. (b) Difference emission spectra of the LMCT state and ferrocyanide with respect to ferricyanide at the $\mathrm{e}_{\mathrm{g}}$ resonance (integrated from 707.6 to $709.4 \mathrm{eV}$ and normalized to the maximum intensity). fluorescence yield (PFY) X-ray absorption spectra of the LMCT state and ferrocyanide, taken as differences with respect to ferricyanide. The PFY spectra are generated from the RIXS difference maps (Figure 1b,c) by integration over energy transfers in the range of $2-7.5 \mathrm{eV}$, thereby isolating the $\mathrm{t}_{2 \mathrm{~g}}^{5} \mathrm{e}_{\mathrm{g}}^{1}$ and $t_{2 \mathrm{~g}}^{5} \mathrm{Lt}_{2 \mathrm{~g}}^{* 1}$ final states discussed above. To enable a qualitative comparison, the PFY difference intensities are normalized to the respective depletion of the ferricyanide $t_{2 g}^{5}$ final state (integrated in the range of 704.9-706.7 eV incidence energy, $-1.2-1.2 \mathrm{eV}$ energy transfer). In the difference PFY of the LMCT state, the onset of the $t_{2 g}^{5} e_{g}^{1}$ final state is at lower incident energy than in ferrocyanide. Such a shift can be interpreted to reflect stabilizing interactions between an increased electron density at the metal site and the corehole, leading to smaller core-excitation energies. This can result from varying metal-ligand covalency, which has previously been observed when comparing $\mathrm{Fe}$ complexes with different ligands. ${ }^{29}$ Of particular interest here is therefore the $t_{2 g}^{5} \mathrm{Lt}_{2 \mathrm{~g}}^{* 1}$ final state intensity in Figure $2 \mathrm{a}$, as it directly probes the strength of $\pi$-back-donation. The feature exhibits similar intensity in the LMCT state and ferrocyanide, which indicates comparable back-donation of Fe $3 \mathrm{~d}$ electron density to the $\mathrm{Lt}_{2 \mathrm{~g}}^{*}$ orbitals for the two species. This is consistent with previous studies, where the $t_{2 g}$ occupation (same for LMCT state and ferrocyanide) was found to mainly govern $\pi$-back-donation. ${ }^{6}$ With comparable $\pi$-back-donation in both species, the overall increase of charge density at the Fe site in the LMCT state relative to ferrocyanide (as deduced from the shift of the PFY onset) can therefore be expected to result from increased $\sigma$ donation, caused by the ligand-hole in the LMCT state. Enhanced electron donation with constant back-donation would strengthen the overall bond between metal and ligand, likely to be reflected in $10 \mathrm{Dq}$. Figure $2 \mathrm{~b}$ shows the intensity of the $\mathrm{t}_{2 \mathrm{~g}}^{5} \mathrm{e}_{\mathrm{g}}^{1}$ final state integrated in the incidence energy range of $707.6-709.4 \mathrm{eV}$ and displayed as a function of the energy transfer. The peak positions of the $t_{2 g}^{5} e_{g}^{1}$ final state on the energy-transfer axis are fitted with Gaussian functions and indeed indicate a rougly $10 \%$ increase of $10 \mathrm{Dq}(\Delta E=(0.3 \pm$ $0.1) \mathrm{eV}$ ) in the LMCT state as compared to ferrocyanide. We emphasize that these trends in both excited-state covalencies and ligand field parameters are directly inferred from timeresolved L-edge RIXS, making it a powerful probe of transient charge distributions of photoexcited metal sites.

To corroborate the interpretations of the observed spectral trends and further characterize the electronic structure of the LMCT state, calculations at the complete active space level $(\mathrm{CASSCF})^{30}$ with second-order perturbation theory $(\mathrm{CASPT} 2)^{31}$ were carried out for ferricyanide, its LMCT state, and ferrocyanide with the Molcas 8.0 program. ${ }^{32}$ Details on the calculations can be found in the Supporting Information. While our previously published restricted active space simulations ${ }^{6,28}$ reproduce the overall RIXS spectral trends of all species, positions and intensities of features which involve $\mathrm{Fe} 2 \mathrm{p} \rightarrow \mathrm{Lt}_{2 \mathrm{~g}}^{*}$ excitation have been found to depend particularly strongly on the computational parameters. ${ }^{33}$ For the most accurate state characterization we therefore resort to single-state calculations that include 13 of the valence orbitals illustrated in Figure 1 in an unbiased complete active space. Because of the severe computational cost scaling of such calculations it is not possible to simultaneously include all six of the $\mathrm{Lt}_{1 \mathrm{u}}$ orbitals as an addition to our previously established ${ }^{28,33}$ valence active space of 10 gerade orbitals. While only three of the $\mathrm{Lt}_{1 \mathrm{u}}$ orbitals are thus directly included 
in the active space, they are allowed to mix with the remaining three through the self-consistent orbital optimization, and all six are correlated through the subsequent multiconfigurational perturbation theory.

To visualize the results of the calculations in a many-electron description, we turn to the charge density difference (CDD) plots displayed in Figure 3a. The orbitals representing the hole

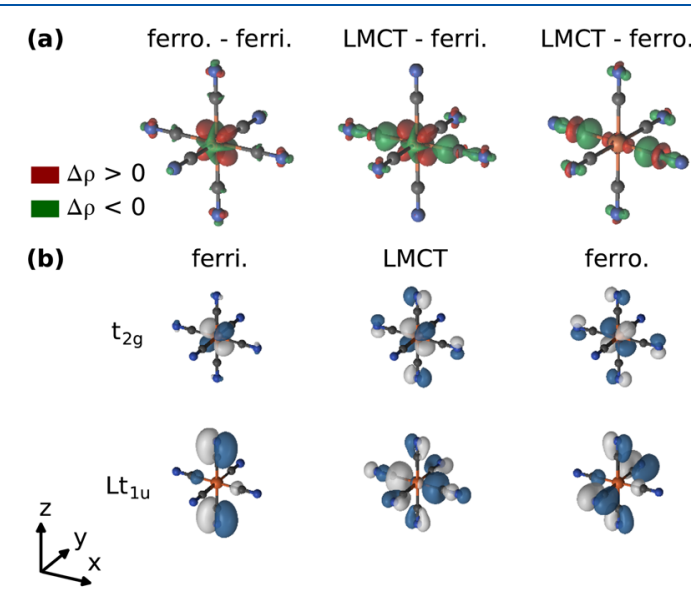

(c)

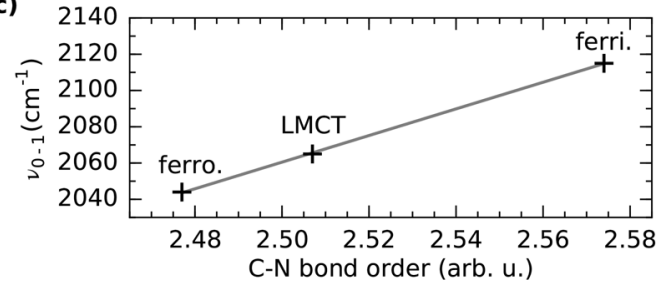

Figure 3. (a) Charge density differences (CDDs) of the LMCT state and ferrocyanide taken with respect to ferricyanide. To isolate ligandhole effects, the CDD of the LMCT state with respect to ferrocyanide is additionally shown. All differences are calculated at the CASPT2 level within the optimized geometry of ferricyanide. (b) Orbitals containing the hole are shown for ferricyanide and the LMCT state. The corresponding active (fully filled) orbitals of ferrocyanide are displayed in addition. (c) Literature values of the $\mathrm{CN}$-stretch frequencies $^{13,25}$ as a function of the calculated average $\mathrm{C}-\mathrm{N}$ bond order.

in ferricyanide $\left(t_{2 g}\right)$ and the LMCT state $\left(\mathrm{Lt}_{1 \mathrm{u}}\right)$ are displayed in Figure $3 \mathrm{~b}$ along with the corresponding (fully filled) orbitals of ferrocyanide. The CDDs are calculated for the optimized structure of ferricyanide, which exhibits a small Jahn-Teller distortion due to the open $t_{2 g}$-shell ${ }^{24,28}$ that leads to the symmetry-reduced $\left(\mathrm{D}_{4 \mathrm{~h}}\right)$ behavior of the individual orbitals. The CDDs clearly confirm the local similarities between ferrocyanide and the LMCT state as experimentally observed from Fe L-edge RIXS. With ferricyanide as a reference (Figure 3a, left and middle), ferrocyanide and the LMCT state both exhibit a distinct increase in charge at the metal center in a shape that resembles the $t_{2 g}$ orbital, hence directly resulting from its increased occupation (Figure $3 \mathrm{~b}$ ). Both species additionally exhibit a decrease in charge even closer to the metal center, which in an indirect manner also results from the increased $t_{2 g}$ occupation. The reduced electron repulsion between metal $3 \mathrm{~d}$ and ligand electron density in ferricyanide allows for stronger electron donation than in ferrocyanide. ${ }^{6,15}$ The same appears to hold in the LMCT state. A quantification of the metal-ligand covalencies is found in Table 1, where the $\mathrm{Fe} 3 \mathrm{~d}$ contents of the $\mathrm{Le}_{\mathrm{g}}$ and $\mathrm{Lt}_{2 \mathrm{~g}}^{*}$ orbitals are presented as
Table 1. Metal-Ligand Covalency as Expressed through the Summed Fe 3d Atomic Orbital Contents of the $\sigma$-Donating Le $_{\mathrm{g}}$ and $\pi$-Accepting $\mathrm{Lt}_{2 \mathrm{~g}}^{*}$ Molecular Orbitals ${ }^{a}$

$\begin{array}{lccc} & \text { ferricyanide } & \text { LMCT state } & \text { ferrocyanide } \\ \mathrm{Le}_{\mathrm{g}} & 22.4 & 15.9 & 14.1 \\ & 24.8 & 19.4 & 14.7 \\ \text { mean } & 23.6 & 17.7 & 14.4 \\ \mathrm{Lt}_{2 \mathrm{~g}}^{*} & 3.8 & 10.7 & 10.6 \\ & 5.9 & 11.1 & 11.0 \\ & 5.9 & 10.7 & 11.0 \\ \text { mean } & 5.2 & 10.8 & 10.9\end{array}$

${ }^{a_{T}}$ The values are presented as percentages normalized to the total contributions of all atomic orbitals to the respective molecular orbital.

they are generally used to measure the strength of $\sigma$-donation and $\pi$-back-donation, respectively. The orbital contents confirm the general trend of reduced $\sigma$-donation and increased $\pi$-back-donation as a result of increased $t_{2 g}$ occupation in both ferrocyanide and the LMCT state.

Similar to the experimental findings, the differences regarding the electronic structure of the LMCT state and ferrocyanide as compared to ferricyanide therefore appear to be mainly determined by the $t_{2 g}$ occupation. Still, smaller differences can also be observed as an effect of the reduced electron density at the ligands in the LMCT state. This can be seen in the CDD of the LMCT state with respect to ferrocyanide (Figure 3a, right), where a charge decrease is present on the ligand $x$-axis, which can be interpreted as the ligand-hole created by the optical excitation. In accordance with previous denotation, ${ }^{6,28}$ the ferricyanide and ferrocyanide $\mathrm{Lt}_{1 \mathrm{u}}$ orbitals included in the active space (i.e., the three out of six most correlated) are of predominantly $\pi$ character (Figure $3 \mathrm{~b}$, left and right). However, the excitation in the LMCT state appears to be from a $\sigma$-dominated $\mathrm{Lt}_{1 \mathrm{u}}$ orbital (Figure $3 \mathrm{~b}$, middle). Consequently, upon optical excitation, the electronhole appears to be localized to the $\sigma$ - rather than to the $\pi$ system, as a result of orbital relaxation within the $\mathrm{Lt}_{1 \mathrm{u}}$ manifold.

The creation of a hole in a $\mathrm{Lt}_{1 \mathrm{u}}$ orbital, which is of strong $\sigma$ character and essentially nonbonding with respect to the $\mathrm{Fe}-\mathrm{C}$ bond, decreases electron density between the $\mathrm{Fe}$ site and the ligand (Figure 3a, right) while not significantly affecting the bond. It instead allows for better overlap between $\mathrm{Fe} 3 \mathrm{~d}$ and $\mathrm{Le}_{\mathrm{g}}$ orbitals. The improved overlap counteracts the effect of lowering the ligand orbital energies, which should make the ligand a poorer $\sigma$-donor. ${ }^{34}$ The result is a net increase in $\sigma$ donation with respect to ferrocyanide (compare Table 1) and thus higher electron density on the metal, which is indeed visible on the CDD between the LMCT state and ferrocyanide (Figure 3a, right). This picture is also consistent with the reduced $\mathrm{Fe}-\mathrm{C}$ bond length in the LMCT state compared to ferrocyanide seen in previous calculations. ${ }^{6,28}$ With constant $\pi$ back-donation, this indicates an overall stronger bonding interaction between metal and the ligands in the LMCT state than in ferrocyanide. We emphasize that this fully confirms the experimental observations of the PFY onset shift to lower energies as well as an increase of $10 \mathrm{Dq}$ in the LMCT state.

Notably, our results are also fully compatible with previous interpretations of steady-state and time-resolved IR-spectroscopy. Shifts of the intraligand IR-stretch frequencies have been shown to typically accompany changes in covalency. This correlation has been established by steady-state IR and 
confirmed by X-ray absorption and RIXS measurements upon comparison of metal cyanides in different oxidation states. ${ }^{6,13,15}$ Figure $3 \mathrm{c}$ shows literature values of the IR-stretch frequencies of ferricyanide, the LMCT state, and ferrocyanide $^{13,25}$ as a function of the $\mathrm{C}-\mathrm{N}$ bond order obtained from our CASPT2 calculations. The bond orders averaged over all ligands scale linearly with the IR-stretch frequencies, thereby accurately reproducing the continuous blue-shift in the order ferrocyanide, LMCT state, and ferricyanide. This agreement therefore confirms the accuracy with which our electronic structure calculations describe the bonding within all three species. While the IR blue-shift of ferricyanide with respect to both other species is then well-explained by the smaller $\pi$-backdonation into the $\mathrm{C}-\mathrm{N}$ antibonding $\mathrm{Lt}_{2 \mathrm{~g}}^{*}$ orbitals, the difference between ferrocyanide and the LMCT state must, because of their similar $\pi$-back-donation, instead result from the ligand-hole. However, it is difficult to break down and attribute the difference to changes in single orbitals with respect to their character and occupation. Further rationalization of the $\mathrm{C}-\mathrm{N}$ bonding is therefore better left for studies with a ligand- rather than metal-centric probe.

In summary, the current work demonstrates that fs RIXS can be applied to derive a detailed metal-centric characterization of transient charge distributions in photoexcited TM complexes in terms of orbital occupation and metal-ligand covalency. Specifically, by studying the LMCT state of ferricyanide we find that $\pi$-back-donation is primarily determined by the metal site occupation, whereas $\sigma$-donation is influenced by the presence of the ligand-hole, a conclusion that underscores the value of steady-state concepts for interpreting the properties of electronic-excited states. The two effects are experimentally disentangled by comparison to steady-state measurements of ferrocyanide but are also fully corroborated by comparison to quantum chemical calculations. We therefore believe that the access to the transient charge distribution provided by timeresolved L-edge RIXS will be generally applicable to a wide range of chemical reactions involving TM complexes. As electronic charge and its deficiency are drivers of chemical reactivity, detailed insight into the transient electronic structure of the catalytic center can aid in understanding and tailoring chemical properties of photoactivated TM complexes.

\section{ASSOCIATED CONTENT}

\section{S Supporting Information}

The Supporting Information is available free of charge on the ACS Publications website at DOI: 10.1021/acs.jpclett.8b01429.

Experimental and computational details (PDF)

\section{AUTHOR INFORMATION}

\section{Corresponding Authors}

*E-mail: odelius@fysik.su.se.

*E-mail: alexander.foehlisch@helmholtz-berlin.de.

\section{ORCID $\odot$}

Raphael M. Jay: 0000-0001-9607-8264

Jesper Norell: 0000-0003-1058-2588

Martin Beye: 0000-0002-3924-2993

Huiyang W. Liang: 0000-0001-6548-2061

Marcus Lundberg: 0000-0002-1312-1202

Philippe Wernet: 0000-0001-7011-9072

Michael Odelius: 0000-0002-7023-2486

\section{Present Addresses}

-A.M.: Elementary Particle Physics Group, Department of Physics, University of Warwick, Coventry CV4 7AL, United Kingdom.

W.Z.: Center for Advanced Quantum Studies, Department of Physics, Beijing Normal University, Beijing 100875, China.

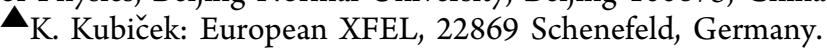

\section{Notes}

The authors declare no competing financial interest.

\section{ACKNOWLEDGMENTS}

R.M.J., S.E., and A.F. acknowledge funding from the ERCADG-2014 - Advanced Investigator Grant - No. 669531 EDAX under the Horizon 2020 EU Framework Program for Research and Innovation. J.N., M.O., S.E., M.B., B.K., W.Q., S.A.T., P.W., and A.F. acknowledge partial or full support by the Helmholtz-Virtual Institute VI419 "Dynamic Pathways in Multidimensional Landscapes”. M.O. and M.L. acknowledge financial support from the Swedish Research Council (VR). W.Z., H.W.L., K. Kunnus, and K.G. acknowledge support from the AMOS program within the Chemical Sciences, Geosciences and Biosciences Division of the Office of Basic Energy Sciences, Office of Science, U.S. Department of Energy. M.B. and K. Kubiček acknowledge financial support from the Volkswagen Foundation under the Peter Paul Ewald fellowship program (87008). S.A.T. acknowledges support from the DFG within the SFB 755 (B03) and SFB 1073 (C02). To 2017, the Max Planck Society is thanked for financial support. M.L. acknowledges support from the Knut and Alice Wallenberg Foundation (Grant No. KAW-2013.0020). Portions of this research were performed on the Soft X-ray Materials Science (SXR) Instrument at the Linac Coherent Light Source (LCLS), a division of SLAC National Accelerator Laboratory and an Office of Science user facility operated by Stanford University for the U.S. Department of Energy. The SXR Instrument is funded by a consortium whose membership includes the LCLS, Stanford University through the Stanford Institute for Materials Energy Sciences (SIMES), Lawrence Berkeley National Laboratory (LBNL), the University of Hamburg through the BMBF priority program FSP 301, and the Center for Free Electron Laser Science (CFEL). The quantum chemical calculations were partially performed on resources provided by the Swedish National Infrastructure for Computing (SNIC) at the HPC2N center. We gratefully acknowledge the support of the LCLS staff and technical support by Christian Weniger as well as Ignacio Fernández Galván for useful discussions.

\section{REFERENCES}

(1) Gray, H. B.; Maverick, A. W. Solar Chemistry of Metal Complexes. Science 1981, 214, 1201-1205.

(2) Chirik, P. J. Iron- and Cobalt-Catalyzed Alkene Hydrogenation: Catalysis with Both Redox-Active and Strong Field Ligands. Acc. Chem. Res. 2015, 48, 1687-1695.

(3) Liu, Y.; Persson, P.; Sundström, V.; Wärnmark, K. Fe N -Heterocyclic Carbene Complexes as Promising Photosensitizers. Acc. Chem. Res. 2016, 49, 1477-1485.

(4) Ament, L. J. P.; van Veenendaal, M.; Devereaux, T. P.; Hill, J. P.; van den Brink, J. Resonant Inelastic X-ray Scattering Studies of Elementary Excitations. Rev. Mod. Phys. 2011, 83, 705-767.

(5) van Schooneveld, M. M.; Gosselink, R. W.; Eggenhuisen, T. M.; Al Samarai, M.; Monney, C.; Zhou, K. J.; Schmitt, T.; de Groot, F. M. F. A Multispectroscopic Study of 3 d Orbitals in Cobalt Carboxylates: 
The High Sensitivity of 2p3d Resonant X-ray Emission Spectroscopy to the Ligand Field. Angew. Chem., Int. Ed. 2013, 52, 1170-1174.

(6) Kunnus, K.; Zhang, W.; Delcey, M. G.; Pinjari, R. V.; Miedema, P. S.; Schreck, S.; Quevedo, W.; Schröder, H.; Föhlisch, A.; Gaffney, K. J.; et al. Viewing the Valence Electronic Structure of Ferric and Ferrous Hexacyanide in Solution from the $\mathrm{Fe}$ and Cyanide Perspectives. J. Phys. Chem. B 2016, 120, 7182-7194.

(7) Liu, B.; Wang, R.-P.; Glass, E. N.; Hill, C. L.; Cuk, T.; Okamoto, J.; Huang, D.-J.; van Schooneveld, M. M.; de Groot, F. M. F. Distorted Tetrahedral Co II in $\mathrm{K} 5 \mathrm{H}[\mathrm{CoW} 12 \mathrm{O} 40$ ] $\times$ x H $2 \mathrm{O}$ Probed by $2 \mathrm{p} 3 \mathrm{~d}$ Resonant Inelastic X-ray Scattering. Inorg. Chem. 2016, 55, 10152-10160.

(8) Hahn, A. W.; Van Kuiken, B. E.; Al Samarai, M.; Atanasov, M.; Weyhermüller, T.; Cui, Y. T.; Miyawaki, J.; Harada, Y.; Nicolaou, A.; DeBeer, S. Measurement of the Ligand Field Spectra of Ferrous and Ferric Iron Chlorides Using 2p3d RIXS. Inorg. Chem. 2017, 56, 8203-8211.

(9) Wernet, P.; Kunnus, K.; Josefsson, I.; Rajkovic, I.; Quevedo, W.; Beye, M.; Schreck, S.; Grübel, S.; Scholz, M.; Nordlund, D.; et al. Orbital-Specific Mapping of the Ligand Exchange Dynamics of $\mathrm{Fe}(\mathrm{CO}) 5$ in Solution. Nature 2015, 520, 78-81.

(10) Kunnus, K.; Josefsson, I.; Rajkovic, I.; Schreck, S.; Quevedo, W.; Beye, M.; Weniger, C.; Grübel, S.; Scholz, M.; Nordlund, D.; et al. Identification of the Dominant Photochemical Pathways and Mechanistic Insights to the Ultrafast Ligand Exchange of $\mathrm{Fe}(\mathrm{CO}) 5$ to $\mathrm{Fe}(\mathrm{CO}) 4 \mathrm{EtOH}$. Struct. Dyn. 2016, 3, 043204.

(11) Kunnus, K.; Josefsson, I.; Rajkovic, I.; Schreck, S.; Quevedo, W.; Beye, M.; Grübel, S.; Scholz, M.; Nordlund, D.; Zhang, W.; et al. Anti-Stokes Resonant X-ray Raman Scattering for Atom Specific and Excited State Selective Dynamics. New J. Phys. 2016, 18, 103011.

(12) Eckert, S.; Norell, J.; Miedema, P. S.; Beye, M.; Fondell, M.; Quevedo, W.; Kennedy, B.; Hantschmann, M.; Pietzsch, A.; Van Kuiken, B. E.; et al. Ultrafast Independent N-H and N-C Bond Deformation Investigated with Resonant Inelastic X-Ray Scattering. Angew. Chem., Int. Ed. 2017, 56, 6088-6092.

(13) Jones, L. H. Nature of Bonding in Metal Cyanide Complexes as Related to Intensity and Frequency of Infrared Absorption Spectra. Inorg. Chem. 1963, 2, 777-780.

(14) Alexander, J. J.; Gray, H. B. Electronic Structures of Hexacyanometalate Complexes. J. Am. Chem. Soc. 1968, 90, 42604271.

(15) Hocking, R. K.; Wasinger, E. C.; de Groot, F. M. F.; Hodgson, K. O.; Hedman, B.; Solomon, E. I. Fe L-Edge XAS Studies of K 4 $[\mathrm{Fe}(\mathrm{CN}) 6]$ and $\mathrm{K} 3[\mathrm{Fe}(\mathrm{CN}) 6]$ : A Direct Probe of Back-Bonding. J. Am. Chem. Soc. 2006, 128, 10442-10451.

(16) Seidel, R.; Thürmer, S.; Moens, J.; Geerlings, P.; Blumberger, J.; Winter, B. Valence Photoemission Spectra of Aqueous $\mathrm{Fe} 2+/ 3+$ and $[\mathrm{Fe}(\mathrm{CN}) 6] 4-/ 3-$ and their Interpretation by DFT Calculations. $J$. Phys. Chem. B 2011, 115, 11671-11677.

(17) Lundberg, M.; Kroll, T.; Debeer, S.; Bergmann, U.; Wilson, S. A.; Glatzel, P.; Nordlund, D.; Hedman, B.; Hodgson, K. O.; Solomon, E. I. Metal-Ligand Covalency of Iron Complexes from HighResolution Resonant Inelastic X-ray Scattering. J. Am. Chem. Soc. 2013, 135, 17121-17134.

(18) Penfold, T. J.; Reinhard, M.; Rittmann-Frank, M. H.; Tavernelli, I.; Rothlisberger, U.; Milne, C. J.; Glatzel, P.; Chergui, M. X-ray Spectroscopic Study of Solvent Effects on the Ferrous and Ferric Hexacyanide Anions. J. Phys. Chem. A 2014, 118, 9411-9418. (19) Prampolini, G.; Yu, P.; Pizzanelli, S.; Cacelli, I.; Yang, F.; Zhao, J.; Wang, J. Structure and Dynamics of Ferrocyanide and Ferricyanide Anions in Water and Heavy Water: An Insight by MD Simulations and 2D IR spectroscopy. J. Phys. Chem. B 2014, 118, 14899-14912.

(20) Ross, M.; Andersen, A.; Fox, Z. W.; Zhang, Y.; Hong, K.; Lee, J.-H.; Cordones, A.; March, A. M.; Doumy, G.; Southworth, S. H.; et al. Comprehensive Experimental and Computational Spectroscopic Study of Hexacyanoferrate Complexes in Water: From Infrared to Xray Wavelengths. J. Phys. Chem. B 2018, 122, 5075-5086.
(21) Zhang, W.; Ji, M.; Sun, Z.; Gaffney, K. J. Dynamics of SolventMediated Electron Localization in Electronically Excited Hexacyanoferrate(III). J. Am. Chem. Soc. 2012, 134, 2581-2588.

(22) Reinhard, M.; Penfold, T. J.; Lima, F. A.; Rittmann, J.; Rittmann-Frank, M. H.; Abela, R.; Tavernelli, I.; Rothlisberger, U.; Milne, C. J.; Chergui, M. Photooxidation and Photoaquation of Iron Hexacyanide in Aqueous Solution: A Picosecond X-ray Absorption Study. Struct. Dyn. 2014, 1, 024901.

(23) Reinhard, M.; Auböck, G.; Besley, N. A.; Clark, I. P.; Greetham, G. M.; Hanson-Heine, M. W. D.; Horvath, R.; Murphy, T. S.; Penfold, T. J.; Towrie, M.; et al. Photoaquation Mechanism of Hexacyanoferrate(II) Ions: Ultrafast 2D UV and Transient Visible and IR Spectroscopies. J. Am. Chem. Soc. 2017, 139, 7335-7347.

(24) Engel, N.; Bokarev, S. I.; Moguilevski, A.; Raheem, A.; AlObaidi, R.; Möhle, T.; Grell, G.; Siefermann, K. R.; Abel, B.; Aziz, S.; et al. Light-Induced Relaxation Dynamics in the Ferricyanide Ion Revisited with Ultrafast XUV Photoelectron Spectroscopy. Phys. Chem. Chem. Phys. 2017, 19, 14248.

(25) Ojeda, J.; Arrell, C. A.; Longetti, L.; Chergui, M.; Helbing, J. Charge-Transfer and Impulsive Electronic-to-Vibrational Energy Conversion in Ferricyanide: Ultrafast Photoelectron and Transient Infrared Studies. Phys. Chem. Chem. Phys. 2017, 19, 17052-17062.

(26) Kunnus, K.; Rajkovic, I.; Schreck, S.; Quevedo, W.; Eckert, S.; Beye, M.; Suljoti, E.; Weniger, C.; Kalus, C.; Grübel, S.; et al. A Setup for Resonant Inelastic Soft X-ray Scattering on Liquids at Free Electron Laser Light Sources. Rev. Sci. Instrum. 2012, 83, 123109.

(27) Schlotter, W. F.; Turner, J. J.; Rowen, M.; Heimann, P.; Holmes, M.; Krupin, O.; Messerschmidt, M.; Moeller, S.; Krzywinski, J.; Soufli, R.; et al. The Soft X-ray Instrument for Materials Studies at the Linac Coherent Light Source X-ray Free-Electron Laser. Rev. Sci. Instrum. 2012, 83, 043107.

(28) Norell, J.; Jay, R. M.; Hantschmann, M.; Eckert, S.; Guo, M.; Gaffney, K. J.; Wernet, P.; Lundberg, M.; Föhlisch, A.; Odelius, M. Fingerprints of Electronic, Spin and Structural Dynamics from Resonant Inelastic Soft X-ray Scattering in Transient Photo-Chemical Species. Phys. Chem. Chem. Phys. 2018, 20, 7243-7253.

(29) Godehusen, K.; Richter, T.; Zimmermann, P.; Wernet, P. Iron L-Edge Absorption Spectroscopy of Iron Pentacarbonyl and Ferrocene in the Gas Phase. J. Phys. Chem. A 2017, 121, 66-72.

(30) Roos, B. O.; Taylor, P. R.; Sigbahn, P. E. A Complete Active Space SCF Method (CASSCF) Using a Density Matrix Formulated Super-CI Approach. Chem. Phys. 1980, 48, 157-173.

(31) Andersson, K.; Malmqvist, P.-Å.; Roos, B. O. Second-Order Perturbation Theory with a Complete Active Space Self-Consistent Field Reference Function. J. Chem. Phys. 1992, 96, 1218-1226.

(32) Aquilante, F.; Autschbach, J.; Carlson, R. K.; Chibotaru, L. F.; Delcey, M. G.; De Vico, L.; Fdez. Galván, I.; Ferré, N.; Frutos, L. M.; Gagliardi, L.; et al. Molcas 8: New Capabilities for Multiconfigurational Quantum Chemical Calculations Across the Periodic Table. J. Comput. Chem. 2016, 37, 506-541.

(33) Pinjari, R. V.; Delcey, M. G.; Guo, M.; Odelius, M.; Lundberg, M. Restricted Active Space Calculations of L-edge X-ray Absorption Spectra: From Molecular Orbitals to Multiplet States. J. Chem. Phys. 2014, 141, 124116.

(34) Shriver, D. F.; Posner, J. Bridge Addition Compounds. III. The Influence of Boron-Containing Lewis Acids on Electronic Spectra, Vibrational Spectra, and Oxidation Potentials of Some Iron-Cynanide Complexes. J. Am. Chem. Soc. 1966, 88, 1672-1677. 